\title{
OPEN Broadband high-resolution molecular spectroscopy with interleaved mid-infrared frequency combs
}

\begin{abstract}
A. V. Muraviev, D. Konnov \& K. L. Vodopyanov ${ }^{凶}$
Traditionally, there has been a trade-off in spectroscopic measurements between high resolution, broadband coverage, and acquisition time. Originally envisioned for precision spectroscopy of the hydrogen atom in the ultraviolet, optical frequency combs are now commonly used for probing molecular ro-vibrational transitions throughout broad spectral bands in the mid-infrared providing superior resolution, speed, and the capability of referencing to the primary frequency standards. Here we demonstrate the acquisition of 2.5 million spectral data points over the continuous wavelength range of 3.17-5.13 $\mu \mathrm{m}$ (frequency span $1200 \mathrm{~cm}^{-1}$, sampling point spacing $13-21 \mathrm{MHz}$ ), via interleaving comb-tooth-resolved spectra acquired with a highly-coherent broadband dual-frequencycomb system based on optical subharmonic generation. With the original comb-line spacing of $115 \mathrm{MHz}$, overlaying eight spectra with gradually shifted comb lines we fully resolve the amplitude and phase spectra of molecules with narrow Doppler lines, such as carbon disulfide $\left(\mathrm{CS}_{2}\right)$ and its three isotopologues.
\end{abstract}

Coherent laser beams in the 3 to $20 \mu \mathrm{m}$ mid-infrared (mid-IR) region provide a unique prospect for sensing molecules through addressing their strongest absorption bands. Thanks to their coherent and broadband nature, optical frequency combs can probe molecular signatures over an extensive (e.g. more than an octave) spectral span simultaneously ${ }^{1,2}$. When one (or several) of the comb teeth is phase locked to a narrow-linewidth reference laser(s), frequency comb spectroscopy can provide the spectral resolution, which is on par with tunable laser spectroscopy-limited only by the absolute comb-tooth linewidth. However, comb spectroscopy has a strong advantage of massive parallelism of data collection and, most importantly, the absolute optical frequency referencing to an accurate external standard (e.g. atomic clock) over the whole spectral span of the comb. Combs with high degree of phase coherence can be used in the dual comb spectroscopy (DCS) - one of the most advanced spectroscopic techniques. In a dual-comb spectrometer, a sensing comb is transmitted through a sample and then multi-heterodyned against a local oscillator (LO) comb which has a repetition rate $f_{r}$ that differs by a small fraction $\Delta f_{r}$ from that of the sensing comb. Compared to classical Fourier transform infrared spectroscopy, DCS demonstrates remarkable improvement of spectral resolution, data acquisition speed, and sensitivity, all at the same time ${ }^{3}$.

With a high degree of mutual coherence between the two combs in a DCS system, it is possible to obtain comb-tooth resolved spectra ${ }^{3-5}$. Using DCS in the near-IR (comb span 1.36-1.69 $\mu \mathrm{m}$ ), Zolot et al. resolved the phase and amplitude of over 400,000 individual comb modes at a mode spacing of $100 \mathrm{MHz}^{5}$. In the mid-IR, by utilizing frequency combs near $3.3 \mu \mathrm{m}$ (spectral span $12 \mathrm{~cm}^{-1}$ ) achieved by applying a nonlinear mixing step to near-IR combs produced by electro-optic modulation (EOM) technique, Yan et al. resolved 1,200 comb lines with the line spacing of $300 \mathrm{MHz}^{6}$. Subsequently, two groups demonstrated comb-tooth resolved spectra over a broad span of frequencies: Ycas et al. resolved 270,000 comb lines between 2.6 and $5.2 \mu \mathrm{m}$ in four overlapping spectral sequences (with mode spacing $200 \mathrm{MHz}$ and a combined frequency span $1800 \mathrm{~cm}^{-1}$ ) , and Muraviev et al. resolved 350,000 comb lines with a finesse of 4,000 within a single frequency comb spanning 3.1-5.5- $\mu \mathrm{m}$ (mode spacing $115 \mathrm{MHz}$, frequency span $1400 \mathrm{~cm}^{-1}$ ) and also demonstrated simultaneous detection of more than 20 molecular species in a mixture of gases ${ }^{8}$.

Once comb teeth are resolved, the spectral resolution is defined by the comb tooth linewidth, which can be orders of magnitude narrower than the comb-line spacing. Then, high-resolution measurements can be 
implemented by interleaving spectra taken with discretely stepped-either comb repetition rate $f_{\mathrm{r}}$ or carrierenvelope offset (CEO) frequency $f_{\text {ceo }}{ }^{2,9-13}$. For example, using a near-IR comb (span of 1.5-1.64 $\mu \mathrm{m}$ ) and a mechanical Fourier transform spectrometer with mode-resolving capability, Rutkowski et al. performed measurements, which yielded 2.4 million sampling points with a step of $20 \mathrm{kHz}$-performed by interleaving spectra with frequency-shifted comb lines ${ }^{14}$. In the mid-IR, Baumann et al. used difference frequency combs near $3.4 \mu \mathrm{m}$ (spectral span $30 \mathrm{~cm}^{-1}$ ), to attain a high-resolution spectrum of methane, by interleaving spectra acquired by shifting the combs by $25 \mathrm{MHz}$-one-quarter of the $100-\mathrm{MHz}$ comb-tooth spacing ${ }^{10}$. Using quantum cascade laser (QCL) combs, Villares et al. performed DCS measurements near $7 \mu \mathrm{m}$ (span $16 \mathrm{~cm}^{-1}$ ) with the sampling point spacing that was improved from the original comb-tooth spacing of $7.5 \mathrm{GHz}$ to $80 \mathrm{MHz}$ by frequency sweeping the combs via QCL current modulation ${ }^{15}$. Similarly, by utilizing dual QCL combs near $8.3 \mu \mathrm{m}$ (span $55 \mathrm{~cm}^{-1}$ ), Gianella et al. achieved an improvement in the sampling point spacing from $9.8 \mathrm{GHz}$ to $30 \mathrm{MHz}$ by sweeping the frequencies of both the sensing and $\mathrm{LO}$ combs via synchronized current modulation ${ }^{16}$. In the two above QCL scenarios the combs were free running with no absolute optical frequency referencing; rather, the frequency scale was calibrated by comparing the spectra with the HITRAN database ${ }^{17}$. Overall, the spectral width of mid-IR measurements with interleaved combs does not exceed $60 \mathrm{~cm}^{-1}$ with one exception of a silicon microresonator comb with a span of 3-3.5 $\mu \mathrm{m}$ that was scanned with a step of $80 \mathrm{MHz}$ via tuning both the pump laser frequency and the cavity resonance. However, the comb lines were scanned over $16 \mathrm{GHz}-$ a small portion of the $127-\mathrm{GHz}$ mode spacing ${ }^{18}$.

One of the challenges of DCS is to get high spectral resolution over a broad bandwidth-required, for example, in applications related to multi-species detection in gas mixtures. To achieve high resolution, one needs long mutual coherence time between the two combs ${ }^{3}$. If the combs are not fully locked, one can track the relative phase drifts and correct for these in real time, at the expense of having two stable continuous-wave reference lasers, as has been demonstrated in the near- $\mathrm{IR}^{19-21}$, or perform phase correction by a posteriori data processing, as has been shown in the $\mathrm{THz}$ and mid-IR ranges $^{22,23}$. However, both of these methods lack the absolute frequency referencing-a setup needs to be calibrated using, for example, a well-known gas absorption feature.

Degenerate (subharmonic) optical parametric oscillators (OPOs) pumped by mode-locked lasers are noteworthy sources of broadband mid-IR frequency $\operatorname{combs}^{24-28}$ and are now used in spectroscopic studies ${ }^{8,29,30}$, random number generation ${ }^{31}$, and in coherent Ising machines ${ }^{32}$. Their key benefits are: low $(\sim 10 \mathrm{~mW})$ oscillation threshold, extremely broad bandwidth, good stability when the cavity is actively locked to resonance, and high conversion efficiency that can exceed $50 \%{ }^{33}$. It has been established that a subharmonic OPO is an ideal coherent frequency divider without any excess phase noise, which rigorously both coherently down-converts and augments the spectrum of the pump frequency comb $b^{34-37}$.

Here we demonstrate, using a highly-coherent subharmonic DCS system, the acquisition of $>2.5$ million spectral data points over the whole (no gaps) spectrum of $3.17-5.13 \mu \mathrm{m}$ (frequency span $1200 \mathrm{~cm}^{-1}, 36 \mathrm{THz}$ ). The sampling density achieved by interleaving comb-line resolved spectra from consecutively shifted combs was sufficient to fully resolve Doppler-broadened absorption bands of several heavy molecules, such as $\mathrm{CS}_{2}$ and OCS, with the main effort focused on $\mathrm{CS}_{2}$. Its choice was motivated (i) by its importance for atmospheric chemistry ${ }^{38}$, astrobiology $y^{39}$, and medical diagnostics ${ }^{40,41}$, and (ii) by the fact that the accurate high-resolution spectroscopic data for $\mathrm{CS}_{2}$ in the mid-IR range were not yet available.

\section{Experiment}

Dual-comb setup. Our dual-comb system used a pair of subharmonic OPOs based on orientation-patterned GaAs (OP-GaAs) crystal as a $\chi^{(2)}$ gain medium, pumped by a highly coherent twin Tm-fiber frequency comb system with a central wavelength of $1.93 \mu \mathrm{m}$, pulse duration of $90 \mathrm{fs}$, repetition rate $f_{r} \approx 115 \mathrm{MHz}$, and the average power of $300 \mathrm{~mW}$ for each laser ${ }^{8}$.

In order to stabilize Tm laser frequency combs, a portion of each laser's output was used to generate a supercontinuum (SC) in a nonlinear silica fiber. While the 1.1- $\mu \mathrm{m}$ and $2.2-\mu \mathrm{m}$ SC components were used to stabilize the CEO frequency via $f-2 f$. interferometry, the component near $1.56 \mu \mathrm{m}$ was utilized to obtain beat notes with a narrow-linewidth reference diode laser from Redfern Integrated Optics (RIO). Each OPO (Fig. 1) had a ring-cavity bow-tie design with low cavity group delay dispersion (GDD) achieved by (i) using low-dispersion mirrors, (ii) a thin (0.5-mm-long) OP-GaAs crystal, and (iii) an intracavity wedge made of $\mathrm{CaF}_{2}$ for GDD compensation ${ }^{8,36}$. The instantaneous spectral coverage of the DCS system was 3.17-5.13 $\mu \mathrm{m}\left(\operatorname{span} 1200 \mathrm{~cm}^{-1}\right)$ at $-10 \mathrm{~dB}$ and $3.08-5.40 \mu \mathrm{m}$ ( $\operatorname{span} 1400 \mathrm{~cm}^{-1}$ ) at $-20 \mathrm{~dB}$ level. The mutual coherence time between the two subharmonic OPOs was previously measured to be as long as $40 \mathrm{~s}^{8}$. Our data acquisition electronics and frequency counters were referenced to $\mathrm{a} \mathrm{Rb}$ atomic clock, which provided $\sim 10^{-10}$ absolute accuracy of the frequency readings.

Spectroscopic measurements with interleaved combs. We used a one-sided DCS configuration (Fig. 1), where only one ('sensing') comb passes through the gas sample. This eliminates the ambiguity of the double-sided scheme, where an absorption profile is probed by both comb lines simultaneously and allows phase spectrum measurement. For absorption measurements we used a multipass gas cell (AMAC-76LW from Aerodyne Research) with 76-m path length and $0.5-\mathrm{L}$ volume. The sensing and LO combs were combined and sent to an infrared detector (InSb from Kolmar, $77 \mathrm{~K}, 60 \mathrm{MHz}$ ) whose output was fed into a 16-bit analogue-to-digital converter (AlazarTech, ATS9626). We used a wedge made of a dielectric $\left(\mathrm{CaF}_{2}\right)$ in the LO channel (Fig. 1) to compensate the dispersion of the multipass cell windows. This did not affect the measured spectrum but made the interferogram sharper, which helped to minimize a jitter in the coherent interferogram averaging process.

First, we demonstrated that an enormous amount of spectral information can be obtained by spectral interleaving over our broadest comb span of 3.17-5.13 $\mu \mathrm{m}$. The repetition rate offset between the two combs was set to $\Delta f_{r}=138.5 \mathrm{~Hz}$, which allowed mapping the whole $1200 \mathrm{~cm}^{-1}$-wide optical spectrum into a radiofrequency 


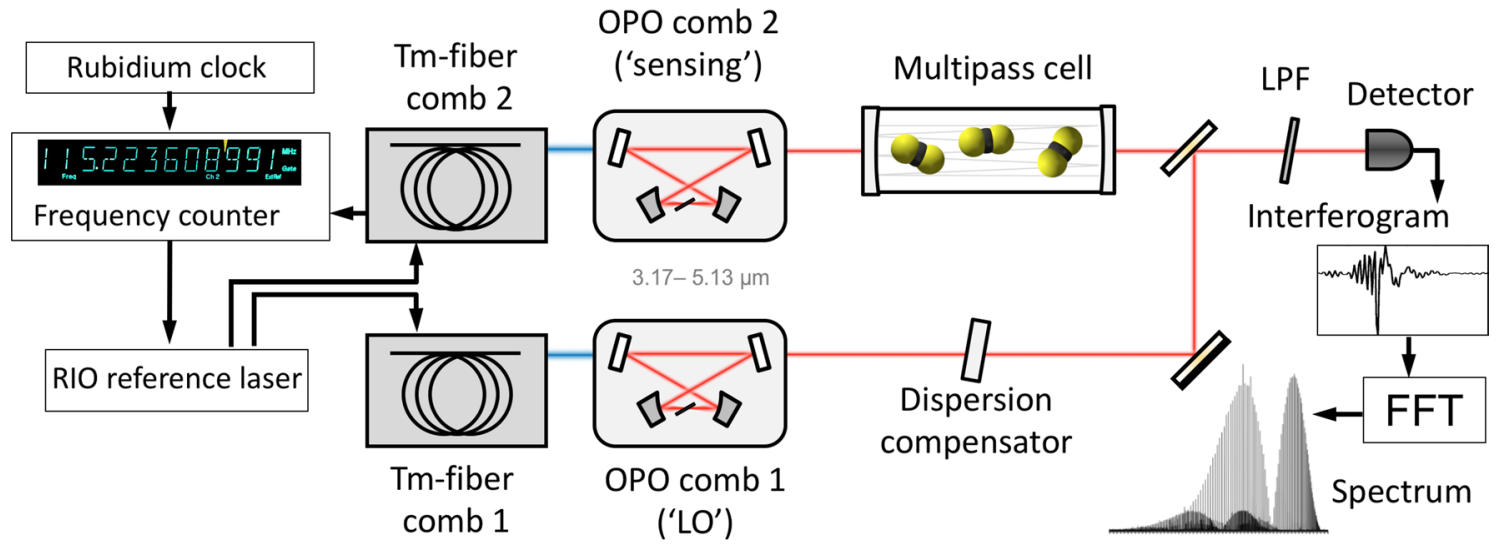

Figure 1. Schematic of the dual-comb spectroscopy setup. LPF, longwave pass filter.

(RF) range of 1-48 MHz ( $<f_{r} / 2$, see Methods). For each comb-line-resolved measurement, we coherently averaged data streams consisting of 10 centerbursts spaced by $1 / \Delta f_{r} \approx 7.2 \mathrm{~ms}$, with the coherent averaging time from a few seconds to several hours. For each data stream, the data acquisition process was triggered by the sharp central spike of the interferogram, which provided good repeatability of the waveforms and allowed long (for at least $>10 \mathrm{~h}$ ) coherent averaging without applying any phase correction procedures. After Fourier transforming the time-domain signal and RF-to-optical frequency up-scaling (see Methods) we obtained a comb-tooth-resolved optical spectrum. By a controlled stepping the optical frequency of the reference RIO laser (see Methods), the comb lines were tuned, thus filling 115-MHz-wide gaps between the comb lines. To fully resolve Doppler-broadened linewidths of species with relatively high molecular weight, such as $\mathrm{CS}_{2}$ (Doppler linewidth $\sim 90 \mathrm{MHz}$ ), we elected to interleave eight comb-line-resolved spectra.

Figure 2a shows the entire comb-tooth-resolved optical spectrum, consisting of more than 2.5 million comb lines obtained from eight interleaved spectra. The total averaging time was $48 \mathrm{~min}$ (6 min for each frequencyshifted measurement). The magnified spectrum, Fig. 2b-d, reveals narrow (Doppler-broadened) absorption dips due to the molecules present in the gas cell $\left(\mathrm{CS}_{2}, \mathrm{CO}\right)$, and Fig. 2e shows phases for individual comb lines, modified by molecular resonances.

The comb-line-resolved spectra were obtained in the following way. A digitized (8-ns point spacing, 16-bit vertical resolution) averaged waveform containing $N_{c}$ centerbursts was resampled to get an integer number of data points $M_{p}$ (typically expressed as a power of 2 ) per time interval that is equal to $1 / \Delta f_{r}$. (In our experiment $\Delta f_{r}$ is known with a fractional accuracy of better than $10^{-9}$, see Methods.) After a Fourier transform, one gets $M_{p} \times N_{c}$ spectral points, where $N_{c}-1$ points out of $N_{c}$ are zero (at the noise level), and only one point is non-zero with its value being proportional to the amplitude of a comb line. This may be seen in the zoomed mode-resolved spectrum of Fig. 2 d (left panel, black color), which was obtained from a waveform containing $N_{c}=10$ centerbursts. As a result of this procedure, the center frequency for each comb line is well-defined with the fractional uncertainty for the absolute value of $7.8 \times 10^{-10}$-through a unique RF-to-optical mapping (see Methods).

\section{Results}

$\mathrm{CS}_{2}$ molecule. For high-precision measurements of the $v_{1}+v_{3}$ band of $\mathrm{CS}_{2}$, our spectral span was narrowed down to approximately $2030-2300 \mathrm{~cm}^{-1}(4.3-4.9 \mu \mathrm{m} ;-10-\mathrm{dB}$ level) - achieved by a combination of tweaking the OPO cavities and using a longpass optical filter (the reduced spectrum is shown in the inset to Fig. $3 \mathrm{a}$ ). The spectral narrowing allowed to increase the signal-to-noise ratio (SNR) ${ }^{11}$ and also increase $\Delta f_{\mathrm{r}}$ by a factor of four, to $\Delta f_{\mathrm{r}}=554 \mathrm{~Hz}$. With the acquisition time of $200 \mathrm{~min}$ for each frequency-shifted spectrum, we reached the fractional standard deviation for the comb-line amplitude of $1.5 \times 10^{-4}\left(\right.$ SNR $\left.6.6 \times 10^{3}\right)$. With the average SNR of $4.4 \times 10^{3}$ over the central $(-4.5-\mathrm{dB}$ level $) \approx 50,000$ modes of the comb (span $\left.\approx 200 \mathrm{~cm}^{-1}\right)$, the DCS figure of merit $(F O M)$, identified in ${ }^{11}$ as $S N R \times M / \sqrt{\tau}$ ( $M$ is the number of modes and is $\tau$ the averaging time), we get $F O M=2.1 \times 10^{6} \mathrm{~Hz}^{1 / 2}$.

The gas mixture in the multipass cell had a total pressure of $6.03 \mathrm{mbar}$ and contained a trace amount of $\mathrm{CS}_{2}$ at a concentration of $94.6 \pm 3 \mathrm{ppm}$ (part-per-million by volume), with $\mathrm{n}$-hexane and room air as buffer gases in similar proportions (see Methods). Figure $3 \mathrm{a}$,b show the absorbance $\left(A=-\ln \left(T / T_{0}\right)\right.$, where $T$ is the power transmission with, and $T_{0}$-without a sample) and phase spectrum corresponding to the $v_{1}+v_{3}$ absorption band of $\mathrm{CS}_{2}$. A magnified view of the $\mathrm{CS}_{2}$ absorbance and phase is given in Figs. 3c,d. The baseline for each interleaved spectrum was obtained by the asymmetric least squares smoothing algorithm based on the Whittaker smoother ${ }^{42}$ with the smoothing parameter that was optimized in such a way that the deviation from the actual data (sufficiently away from a line center) did not exceed the local noise level. Due to the low pressure and high resolution, the spectral lines of other molecules $-\mathrm{H}_{2} \mathrm{O}, \mathrm{CO}_{2}, \mathrm{CO}$, and $\mathrm{N}_{2} \mathrm{O}-$ present in the gas mixture due to the room air, practically didn't interfere with the $\mathrm{CS}_{2}$ lines. On the other hand, $\mathrm{n}$-hexane, used as a solvent for $\mathrm{CS}_{2}$, does not have any characteristic absorption features across the spectral span used here, and gave a very smooth absorption offset. The spectra were obtained from eight interleaved comb-line resolved measurements. Every data point represents a comb line with a known absolute frequency (see Methods). The distance between the 

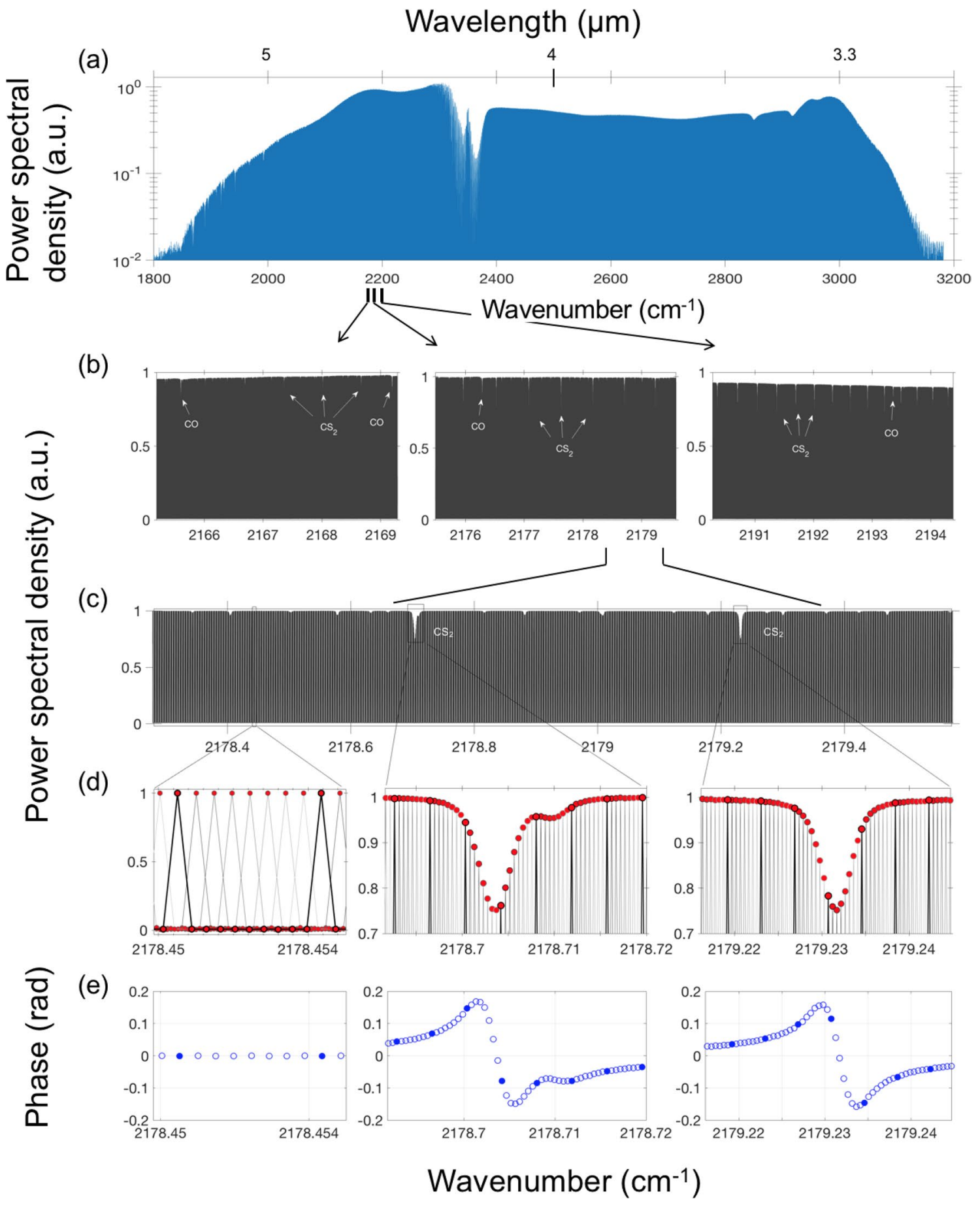

Figure 2. (a) Combined mode-resolved spectrum (log-scale) containing $>2.5$ million comb lines $(-10$ - $\mathrm{dB}$ level), obtained by eight interleaved measurements. The broad dips in the spectrum are due to the atmospheric absorption $\left(\mathrm{H}_{2} \mathrm{O}, \mathrm{CO}_{2}\right)$ outside the gas cell. $(\mathbf{b}, \mathbf{c})$ Magnified view of the spectrum showing narrow features due to molecular absorption inside the cell. (d,e) Further magnification shows amplitudes and phases for the individual interleaved comb lines spaced by $14.4 \mathrm{MHz}$. Features shown in black in (d) and filled circles in (e) correspond to the original comb-line spacing of $115 \mathrm{MHz}$.

sampling points is $14.4 \mathrm{MHz}$-about $1 / 8$ of the original comb-line spacing ( $115 \mathrm{MHz}$ - appears to be adequate for resolving the $\mathrm{CS}_{2}$ Doppler broadened absorption lines.

Figure 4 shows the absorbance spectrum of $\mathrm{CS}_{2}$ plotted on a log-scale that allows to see the weaker bands. For better visibility of the $\mathrm{CS}_{2}$ spectrum, the lines of $\mathrm{CO}$ and $\mathrm{N}_{2} \mathrm{O}$ present in the mixture due to the room air were digitally subtracted via HITRAN simulations using concentration as the only fitting parameter. This was also used to verify the absolute accuracy of our frequency readings: the positions of the $\mathrm{CO}$ and $\mathrm{N}_{2} \mathrm{O}$ peaks deviated from those obtained from HITRAN by less than $1 \mathrm{MHz}$ and allowed clean removal of these peaks. Additionally, 
(a)

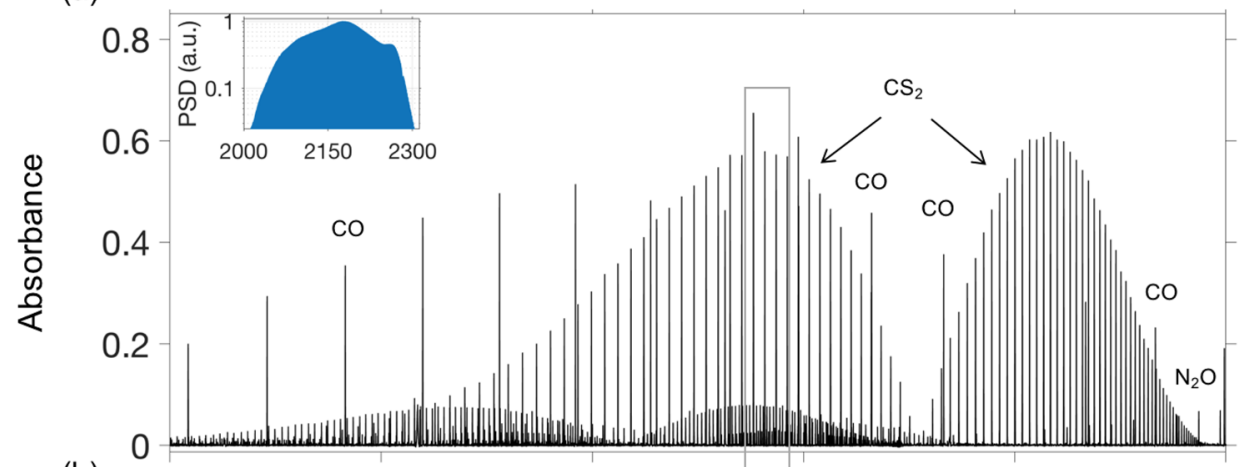

(b)

0.2

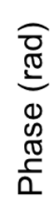

(c)

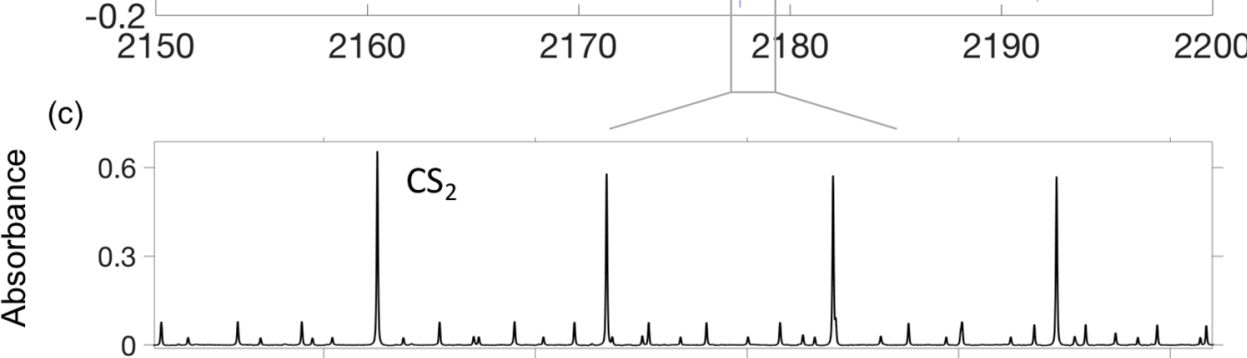

$\frac{0}{0}$
0
0
$\frac{0}{0}$
$\frac{\pi}{2}$

d) 0.2

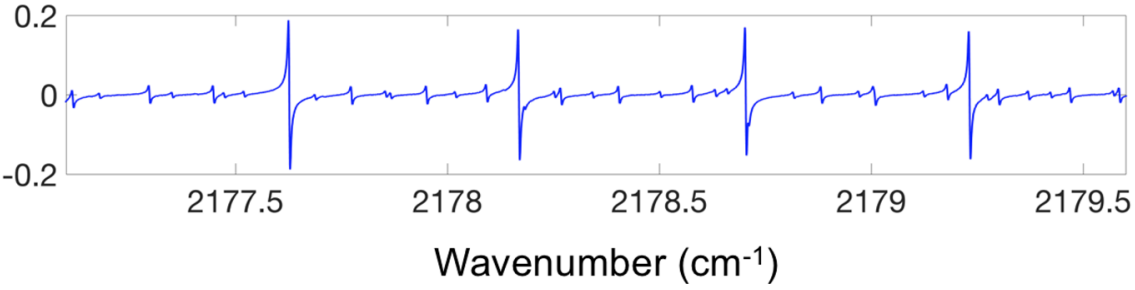

Figure 3. (a,b) Absorbance $\left(=-\ln \left(T / T_{0}\right)\right)$ and phase spectra for the $v_{1}+v_{3}$ band of $\mathrm{CS}_{2}$ obtained at 14.4$\mathrm{MHz}$ spectral point spacing. (c,d) Magnified view of the $\mathrm{CS}_{2}$ absorbance and phase. The spectral peaks of $\mathrm{CO}$ and $\mathrm{N}_{2} \mathrm{O}$ in (a,b) are due to the room air in the cell. Inset: Reduced comb spectrum (log-scale) used in this experiment. PSD, power spectral density.

few slices between 2135 and $2165 \mathrm{~cm}^{-1}$ — corresponding to strong peaks of water vapor outside the gas cell-were manually cut out in Fig. 4.

The high resolution and long coherent averaging allowed us to identify 981 spectral lines of the $v_{1}+v_{3}$ absorption band of $\mathrm{CS}_{2}$. In fact, because of the low noise of the baseline, we were able to detect $\mathrm{CS}_{2}$ lines that were about $10^{3}$ times weaker than the strongest ones (see the inset to Fig. 4). In addition to the main molecule, we were able to resolve the spectra of three isotopologues $\left({ }^{34} \mathrm{~S}^{12} \mathrm{C}^{32} \mathrm{~S},{ }^{33} \mathrm{~S}^{12} \mathrm{C}^{32} \mathrm{~S}\right.$, and ${ }^{32} \mathrm{~S}^{13} \mathrm{C}^{32} \mathrm{~S}$ ), as well as the spectra of seven hot bands due to transitions from the thermally excited vibrational states, labelled in Fig. 4 . The inset shows the two peaks corresponding to the low-abundance ${ }^{33} \mathrm{~S}$ isotope $\left(\sim 0.8 \%\right.$ with respect to $\left.{ }^{32} \mathrm{~S}\right)$, along with the two peaks for the main molecule. More details on our $\mathrm{CS}_{2}$ measurements, including line lists and the interpretation of the bands can be found in ${ }^{43}$.

OCS molecule. Figure 5 presents the portion of the spectrum (absorbance and phase) from the same experiment that reveals the presence of trace amounts of carbonyl sulfide (OCS) molecule, although this molecule was not originally present in the gas mixture. The plot also shows the simulated (HITRAN) spectrum for the asymmetric $v_{3}$ stretch of OCS. From the known absorption cross section of OCS, its concertation was evaluated to be $1.75 \mathrm{ppm}$. Our repeated measurements revealed that the OCS concertation kept rising at a rate of approximately $15 \%$ per day, while the concertation of $\mathrm{CS}_{2}$ was decreasing at a rate of $0.5-0.7 \%$ per day. We hypothesize that the emergence of OCS is due to a chemical oxidation reaction of $\mathrm{CS}_{2}$ in the presence of $\mathrm{O}_{2}$ in the gas cell ${ }^{44}$. 


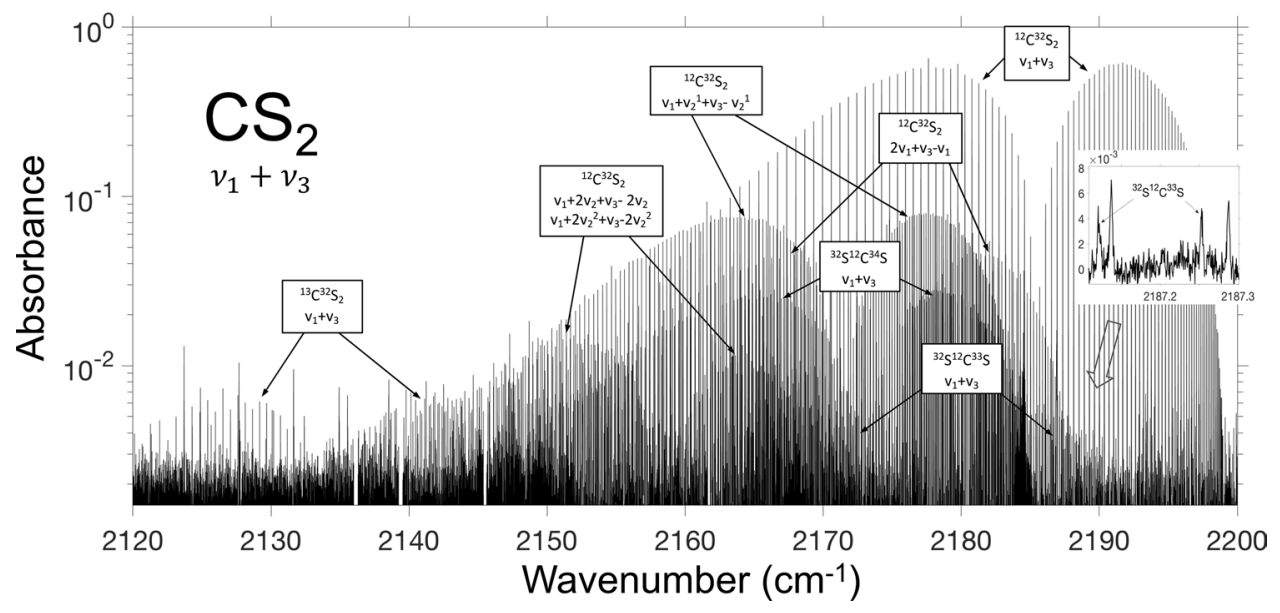

Figure 4. Log-scale spectrum corresponding to the $v_{1}+v_{3}$ band of $\mathrm{CS}_{2}$ that include: bands due to isotopes: ${ }^{34} \mathrm{~S}$, ${ }^{33} \mathrm{~S}$, and ${ }^{13} \mathrm{C}$ and hot bands from the thermally excited vibrational states $v_{1}$ and $v_{2}$. The inset shows two peaks due to the low-abundance ${ }^{33} \mathrm{~S}$ isotope. For better visibility of the spectrum, the $\mathrm{CO}$ and $\mathrm{N}_{2} \mathrm{O}$ lines were digitally subtracted via HITRAN-based simulations. Also, few slices between 2135 and $2165 \mathrm{~cm}^{-1}$ corresponding to strong water absorption outside the gas cell were manually cut out.

(a)

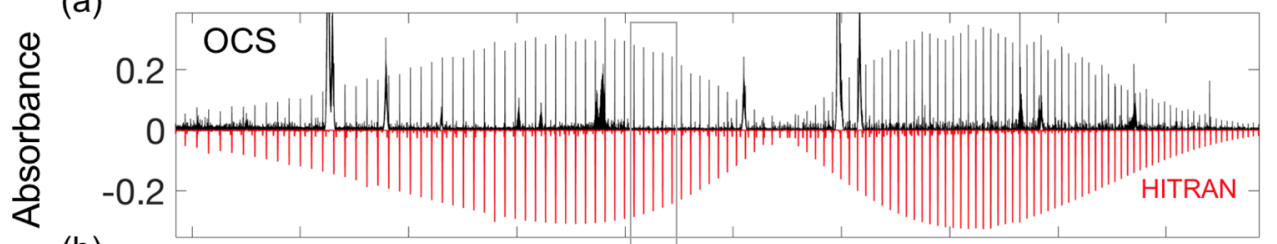

(b)

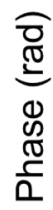

(c)
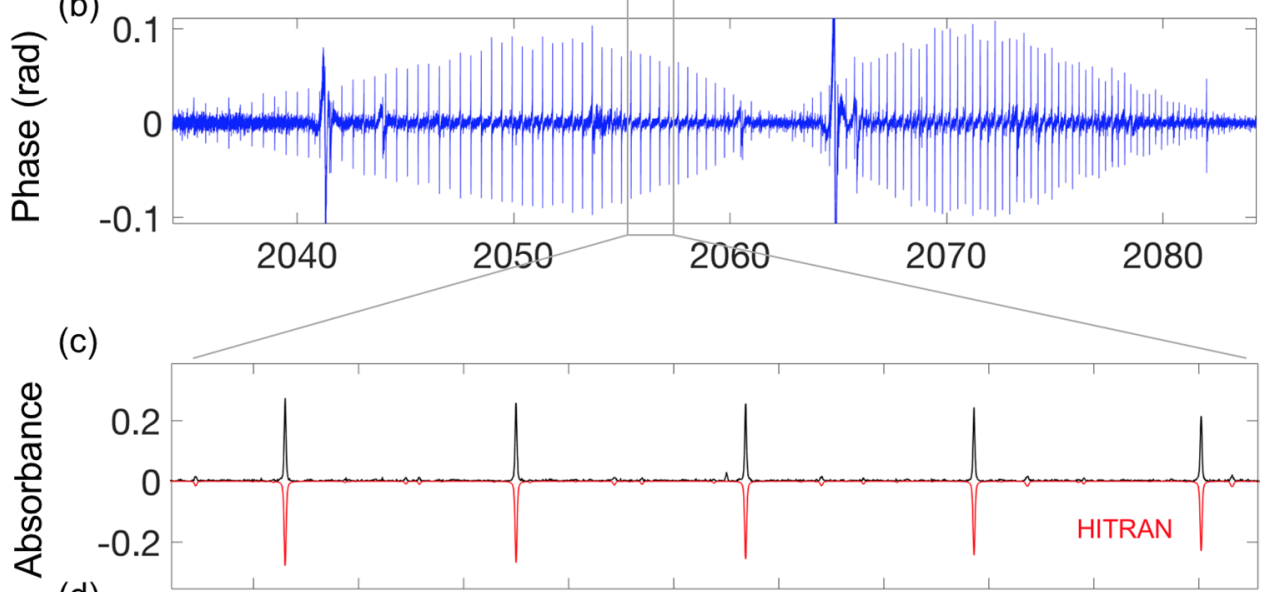

(d)

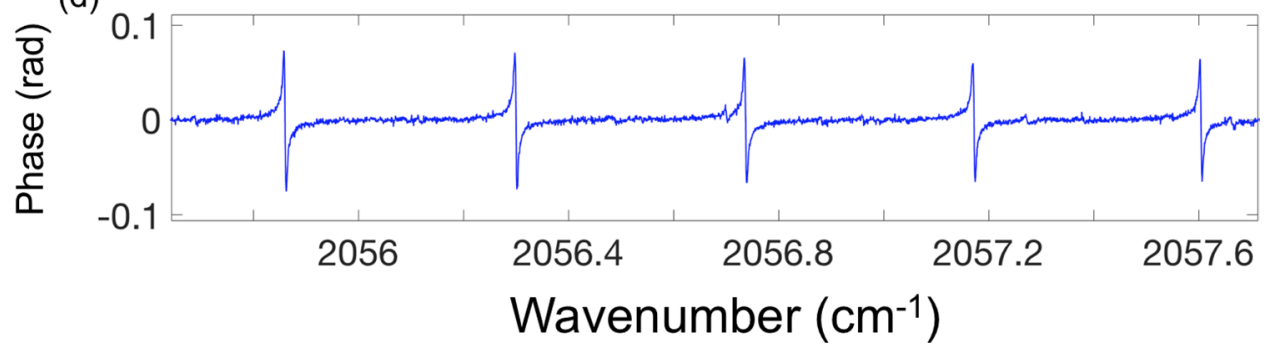

Figure 5. (a) Absorbance and (b) phase spectrum for the $v_{3}$ asymmetric band of OCS. The noise features (e.g. at 2041, $2065 \mathrm{~cm}^{-1}$ etc.) are related to strong (and broad) water absorption lines outside the gas cell. (c,d) Expanded OCS spectrum for the absorbance and phase. Shown in $(\mathbf{a}, \mathbf{c})$ are simulated HITRAN-based absorbance spectra, inverted for clarity. 


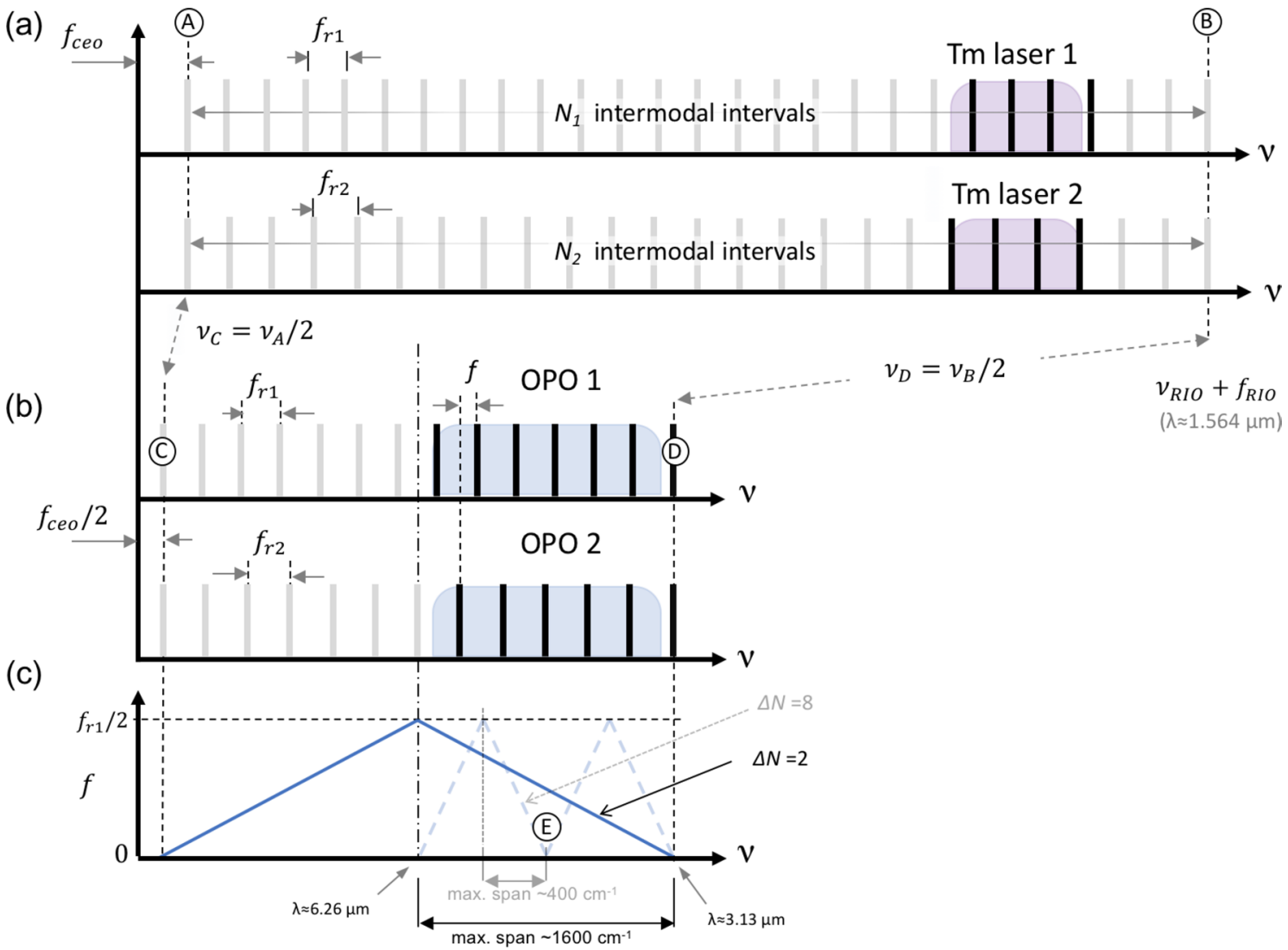

Figure 6. (a) Comb lines extrapolated to zero optical frequency $v$ for the two Tm-fiber pump lasers, with the repetition rates $f_{\mathrm{r} 1}$ and $f_{\mathrm{r} 2}$ respectively. The Tm combs are $f_{c e o}$ stabilized and phase locked to a common narrowband 'RIO' laser $(\lambda \approx 1.564 \mu \mathrm{m})$ so that their teeth overlap at the two lock points 'A' and 'B'. (b) The subharmonic OPO combs are phase locked to the pump and their teeth (e.g. if $\Delta N=2$ and $N_{1,2}$ are even) overlap at the anchor point ' $C$ ' distanced by $f_{\text {ceo }} / 2$ from zero, and at a common anchor point ' $\mathrm{D}$ ' $(\lambda \approx 3.13 \mu \mathrm{m})$. (c) RF-to-optical frequency mapping for $\Delta N=2$ and $\Delta N=8$.

\section{Discussion}

Using a highly-coherent and broadband dual-comb spectroscopy system based on subharmonic generation, we demonstrate the acquisition of up to 2.5 million spectral elements over the wavelength range of 3.17-5.13 $\mu \mathrm{m}$ (frequency span $1200 \mathrm{~cm}^{-1}$ ) - by interleaving spectra with discretely stepped comb-line spacing. Among other molecular absorption features, we fully resolved the Doppler-broadened spectra corresponding to the strongest 981 transitions of the $v_{1}+v_{3}$ band $(4.5-4.7 \mu \mathrm{m})$ of $\mathrm{CS}_{2}$ and its three isotopologues with $\sim 50-\mathrm{kHz}$ accuracy of the absolute frequency referencing. Most of the line intensities were accurately measured for the first time and will be included in the line lists for molecular databases ${ }^{43}$. Furthermore, the obtained data can be used as a testing ground for theoretical models. The sampling point spacing in our experiment can be further reduced (at the expense of the measurement time) by 2-3 orders of magnitude, limited only by the $50-\mathrm{kHz}$ uncertainty in the absolute frequency. This in turn can be further improved using a more stable narrow-linewidth optical reference laser and a tighter feedback loop.

\section{Methods}

OPO comb modes and RF-to-optical frequency mapping. The comb modes for both Tm-fiber pump lasers were locked, at the two common anchor points (Fig. 6): near zero frequency with a common carrierenvelope offset (CEO) $f_{\text {ceo }}^{\text {pump }}=+190 \mathrm{MHz}$ (point A), and near the frequency of a narrow-linewidth $(\sim 3 \mathrm{kHz})$ continuous wave $(\mathrm{CW})$ reference 'RIO' semiconductor laser at near $\lambda \approx 1564 \mathrm{~nm}$ with an offset $f_{R I O}=+140 \mathrm{MHz}$ (point B). Between $\mathrm{A}$ and $\mathrm{B}$, there are $N_{1}$ intermodal intervals $\left(f_{\mathrm{r} 1}\right)$ for laser 1 and $N_{2}$ intervals $\left(f_{\mathrm{r} 2}>f_{\mathrm{r} 1}\right)$ for laser 2 , such that $N_{1} \times f_{\mathrm{r} 1}=N_{2} \times f r_{2}$ and

$$
\Delta f_{r}=f_{r 2}-f_{r 1}=\frac{N_{1}-N_{2}}{N_{2}} f_{r 1}=\frac{\Delta N}{N_{2}} f_{r 1}=\frac{\Delta N}{N_{1}} f_{r 2} .
$$

The repetition rate offset $\Delta f_{r}$ between the two lasers is quantized. For example, for $\Delta N=2, \Delta f_{\mathrm{r}} \approx 138.5 \mathrm{~Hz}$ and for even $N_{1,2}$ such that $N_{1}=2\left(k_{0}+1\right)$ and $N_{2}=2 k_{0}$ with $k_{0}$ an integer, the ratios $f_{\mathrm{r} 1} / \Delta f_{\mathrm{r}}$ and $f_{\mathrm{r} 2} / \Delta f_{\mathrm{r}}$ are integer numbers. In fact, in our experiments the measured ratio $f_{\mathrm{r} 1} / \Delta f_{\text {rep }}\left(\approx k_{0}\right)$ was an integer: 831,844 with a remainder after division $<10^{-3}$. 
Both OPO combs were running in the frequency-divide-by-2 mode with respect to the pump, such that that $\mathrm{CEO}$ frequency for both of them (anchor point $\mathrm{C}$ in Fig. 6 was half of that of the pump CEO:

$$
f_{\text {ceo }}^{O P O 1}=f_{\text {ceo }}^{\mathrm{OPO} 2}=\frac{1}{2} f_{\text {ceo }}^{\text {pump }} .
$$

Another anchor point (D) is at $v_{\mathrm{D}}=1 / 2 v_{\mathrm{B}}$ (near $\sim 3.13 \mu \mathrm{m}$ ), just above (in frequency) our broadest spectral span. For $\Delta N=2$ we used the following RF-to-optical mapping for the sensing comb:

$$
v=v_{D}-\frac{f_{r 2}}{\Delta f_{r}} f=\frac{1}{2} f_{c e o}^{\text {pump }}+\frac{f_{r 2}}{\Delta f_{r}}\left(f_{r 1}-f\right),
$$

where $v$ is the optical frequency that interrogates the sample, and $f$ is RF frequency. The whole $1200 \mathrm{~cm}^{-1}$ spectral span is thus mapped to the $1-48-\mathrm{MHz} R$ F range. In a similar way, with the reduced $\left(2030-2300 \mathrm{~cm}^{-1}\right)$ frequency coverage, selected for the study of $\mathrm{CS}_{2}$ molecule, we have used $\Delta N=8, \Delta f_{r}=554 \mathrm{~Hz}$, such that the RF comb's span matches the aliasing free bandwidth, with the following RF-to-optical frequency mapping for the sensing comb:

$$
v=\frac{1}{2} f_{\text {ceo }}^{\text {pump }}+\frac{f_{r 2}}{\Delta f_{r}}\left(3 f_{r 1}-f\right) .
$$

Here, the term $\frac{1}{2} f_{\text {ceo }}^{\text {pump }}+3 \frac{f_{r 1} f_{r 2}}{\Delta f_{r}}$ corresponds to the anchor point "E" in Fig. $6 c$ for both OPO combs, and $\frac{f_{r 2}}{\Delta f_{r}}$ is the RF-to-optical upscaling factor (with a negative sign).

Remarkably, with the known $\Delta N$, frequency offsets $f_{c e o}^{\text {pump }}$ and $f_{R I O}$, and with just two readings from the frequency counters $\left(f_{r 1}\right.$ and $\left.\Delta f_{r}\right)$, we were able to determine the vacuum wavelength of the RIO laser with the fractional accuracy of better than $10^{-9}$.

Stabilization and stepping of the RIO laser and the absolute frequency referencing. Since the two pump Tm-fiber combs are phase locked to a common optical reference (RIO laser), and the OPO combs, in turn, are phase-locked to the Tm combs, the drifts of the RIO laser frequency $\left(v_{R I O}\right)$, typically $\sim 10 \mathrm{MHz}$ during a day, affect the absolute position of the comb lines. To avoid these drifts, we implemented an active $v_{R I O}$ stabilization. Using the readings of a frequency counter referenced to a Rb clock for the repetition rate $\left(f_{r 1}\right)$ of one of the Tm-fiber combs, and applying the difference between the measured and a desired repetition rate as a feedback to act on the RIO laser temperature with a slow servo loop with an update time of $4 \mathrm{~s}$, we achieved the standard deviation for $f_{r 1}$ of $0.09 \mathrm{~Hz}\left(\sigma f_{r 1} / f_{r 1}=7.8 \times 10^{-10}\right)$, which corresponds to the standard deviation of the RIO laser frequency $\sigma v_{R I O}=v_{R I O} \times \sigma f_{r 1} / f_{r 1} \approx 150 \mathrm{kHz}$. Since both OPO combs are pinned to a common frequency offset near zero, the frequency uncertainty of a comb line is proportional to its absolute frequency. For example, for the $\mathrm{CS}_{2}$ absorption band near 4.6- $\mu \mathrm{m}$, the standard deviation is $\sigma v=v \times 7.8 \times 10^{-10} \approx 50 \mathrm{kHz}$.

The interleaved DCS spectra were acquired by stepping, through temperature control, $v_{R I O}$ in eight $42.35-\mathrm{MHz}$ increments (corresponding to $f_{r 1}$ steps of $\approx 25 \mathrm{~Hz}$ ), resulting in the comb-line shifts that vary from $21 \mathrm{MHz}$ (at $\approx 3.2 \mu \mathrm{m}$ ) to $13 \mathrm{MHz}$ (at $\approx 5.1 \mu \mathrm{m}$ ), and were $14.4 \mathrm{MHz}$ (about $1 / 8$ of the original 115-MHz comb-line spacing) near the 4.6- $\mu \mathrm{m}$ absorption band of CS2.

$\mathrm{CS}_{2}$ sample preparation. A droplet of liquid-phase $\mathrm{CS}_{2}$ dissolved in $\mathrm{n}$-hexane at a concentration of $100.6 \mu \mathrm{g} / \mathrm{ml} \pm 2 \%$ (Chem Services, Inc.) was placed in a 10 -ml-volume chemical flask and fully evaporated, together with the ambient room air in the headspace, into the evacuated 0.5 -L multipass gas cell. The resulting pressure in the gas cell was then measured with an accuracy of $\pm 1 \%$. Next, the same procedure was repeated with the chemical flask filled just with room air. The fractional (by molecules) concentration of the hexane- $\mathrm{CS}_{2}$ mixture in the gas cell was determined by comparing the measured pressure in the gas cell due to the mixture (liquid droplet plus air) and the pressure produced due to the flask filled just with air. (In fact, there were comparable concentrations of molecules coming from the droplet and room air.) For spectral measurements, the gas cell was further evacuated to get the total pressure of 6.03 mbar, with the calculated $\mathrm{CS}_{2}$ concentration of $94.6 \mathrm{ppm} \pm 3 \%$ (part-per-million by volume). The gas cell temperature was kept at $293.7 \pm 0.5 \mathrm{~K}$.

Received: 2 July 2020; Accepted: 12 October 2020

Published online: 29 October 2020

\section{References}

1. Schliesser, A., Picqué, N. \& Hänsch, T. W. Mid-infrared frequency combs. Nat. Photon. 6, 440 (2012).

2. Picqué, N. \& Hänsch, T. W. Frequency comb spectroscopy. Nat. Photon. 13, 146-157 (2019).

3. Coddington, I., Swann, W. C. \& Newbury, N. R. Coherent multiheterodyne spectroscopy using stabilized optical frequency combs. Phys. Rev. Lett 100, 013902 (2008).

4. Coddington, I., Swann, W. C. \& Newbury, N. R. Time-domain spectroscopy of molecular free-induction decay in the infrared. Opt. Lett. 35, 1395-1397 (2010).

5. Zolot, M. et al. Direct-comb molecular spectroscopy with accurate, resolved comb teeth over 43 THz. Opt. Lett. 37, 638-640 (2012).

6. Yan, M. et al. Mid-infrared dual-comb spectroscopy with electro-optic modulators. Light Sci. Appl. 6, e17076 (2017).

7. Ycas, G. et al. High-coherence mid-infrared dual-comb spectroscopy spanning 2.6 to $5.2 \mu \mathrm{m}$. Nat. Photon. 12, 202-209 (2018).

8. Muraviev, V., Smolski, V. O., Loparo, Z. E. \& Vodopyanov, K. L. Massively parallel sensing of trace molecules and their isotopologues with broadband subharmonic mid-infrared frequency combs. Nat. Photon. 12, 209-214 (2018).

9. Schiller, S. Spectrometry with frequency combs. Opt. Lett. 27, 766 (2002).

10. Baumann, E. et al. Spectroscopy of the methane $v_{3}$ band with an accurate midinfrared coherent dual-comb spectrometer. Phys. Rev. A 84, 062513 (2011). 
11. Coddington, A., Newbury, N. \& Swann, W. Dual-comb spectroscopy. Optica 3, 414-426 (2016).

12. Weichman, M. L. et al. Broadband molecular spectroscopy with optical frequency combs. J. Molec. Spectrosc. 355, 66-78 (2019).

13. Jacquet, P., Mandon, J., Bernhardt, B., Holzwarth, R., Guelachvili, G., Hänsch, T. W. \& Picqué, N. Frequency comb Fourier transform spectroscopy with kHz optical resolution, in Advances in Imaging, OSA Technical Digest (Optical Society of America, 2009), paper FMB2.

14. Rutkowski, L. et al. Sensitive and broadband measurement of dispersion in a cavity using a Fourier transform spectrometer with $\mathrm{kHz}$ resolution. Opt. Express 25, 21711 (2017).

15. Villares, G., Hugi, A., Blaser, S. \& Faist, J. Dual-comb spectroscopy based on quantum-cascade-laser frequency combs. Nat. Commun. 5, 5192 (2014).

16. Gianella, M., Nataraj, A., Tuzson, B., Jouy, P., Kapsalidis, F., Beck, M., Mangold, M., Hugi, A., Faist, J., \& Emmenegger, L. Highresolution and gapless dual comb spectroscopy with current-tuned quantum cascade lasers. arXiv:1910.13973 (2019).

17. Rothman, L. S. et al. The HITRAN 2008 molecular spectroscopic database. J. Quant. Spectrosc. Radiat. Transf. 110, 533-572 (2009).

18. Yu, M., Okawachi, Y., Griffith, A. G., Lipson, M. \& Gaeta, A. L. Microresonator-based high-resolution gas spectroscopy. Opt. Lett. 42, 4442 (2017).

19. Ideguchi, T., Poisson, A., Guelachvili, G., Picqué, N. \& Hänsch, T. W. Adaptive real-time dual-comb spectroscopy. Nat. Commun. 5, 3375 (2014).

20. Roy, J., Deschênes, J.-D., Potvin, S. \& Genest, J. Continuous real-time correction and averaging for frequency comb interferometry. Opt. Express 20, 21932-21939 (2012).

21. Guay, P. et al. Toward free-running operation of dual-comb fiber lasers for methane sensing. Appl. Opt. 59, B35 (2020).

22. Burghoff, D., Yang, Y. \& Hu, Q. Computational multiheterodyne spectroscopy. Sci. Adv. 2(11), e1601227 (2016).

23. Sterczewski, L. A. et al. Multiheterodyne spectroscopy using interband cascade lasers. Opt. Eng. 57(1), 011014 (2017).

24. Vodopyanov, K. L., Wong, S. T. \& Byer, R. L. Infrared frequency comb methods, arrangements and applications. U.S. patent $8,384,990$ (February 26, 2013).

25. Leindecker, N., Marandi, A., Byer, R. L. \& Vodopyanov, K. L. Broadband degenerate OPO for mid-infrared frequency comb generation. Opt. Express 19, 6304-6310 (2011).

26. Leindecker, N. et al. Octave-spanning ultrafast OPO with 2.6-6.1 $\mu \mathrm{m}$ instantaneous bandwidth pumped by femtosecond Tm-fiber laser. Opt. Express 20, 7047-7053 (2012).

27. Ru, Q. et al. Self-referenced octave-wide subharmonic GaP optical parametric oscillator centered at $3 \mu \mathrm{m}$ and pumped by an Erfiber laser. Opt. Lett. 42, 4756-4759 (2017).

28. Smolski, V. et al. Half-Watt average power femtosecond source spanning 3-8 $\mu \mathrm{m}$ based on subharmonic generation in GaAs. Appl. Phys. B 124, 101 (2018).

29. Vainio and Karhu. Fully stabilized mid-infrared frequency comb for high-precision molecular spectroscopy. Opt. Express 25, 4190 (2017).

30. Loparo, Z. E., Ninnemann, E., Ru, Q., Vodopyanov, K. L. \& Vasu, S. S. Broadband mid-infrared optical parametric oscillator for dynamic high-temperature multi-species measurements in reacting systems. Opt. Lett. 45, 491-494 (2020).

31 Marandi, A., Leindecker, N. C., Vodopyanov, K. L. \& Byer, R. L. All-optical quantum random bit generation from intrinsically binary phase of parametric oscillators. Opt. Express 20, 19322-19330 (2012).

32. Marandi, A., Wang, Z., Takata, K., Byer, R. L. \& Yamamoto, Y. Network of time-multiplexed optical parametric oscillators as a coherent Ising machine. Nat. Photon. 8, 937-942 (2014).

33. Marandi, A., Ingold, K. A., Jankowski, M. \& Byer, R. L. Cascaded half-harmonic generation of femtosecond frequency combs in the mid-infrared. Optica 3, 324-327 (2016).

34. Marandi, A., Leindecker, N., Pervak, V., Byer, R. L. \& Vodopyanov, K. L. Coherence properties of a broadband femtosecond mid-IR optical parametric oscillator operating at degeneracy. Opt. Express 20, 7255-7262 (2012).

35. Lee, K. F. et al. Midinfrared frequency combs from coherent supercontinuum in chalcogenide and optical parametric oscillation. Opt. Lett. 39, 2056-2059 (2014).

36. Smolski, V. O., Yang, H., Gorelov, S. D., Schunemann, P. G. \& Vodopyanov, K. L. Coherence properties of a 2.6-7.5 $\mu$ m frequency comb produced as a subharmonic of a Tm-fiber laser. Opt. Lett. 41, 1388 (2016).

37. Wan, C., Li, P., Ruehl, A. \& Hartl, I. Coherent frequency division with a degenerate synchronously pumped optical parametric oscillator. Opt. Lett. 43, 1059 (2018).

38. .Yang, C., Deng, H., Qian, Y., Li, M., Chen, B., Xu, Z., \& Kan, R. Absorption lines measurements of carbon disulfide at $4.6 \mu \mathrm{m}$ with quantum cascade laser absorption spectroscopy. Spectrochim. Acta 225, 117478 (2020).

39. Landis, G. A. Astrobiology: the case for Venus. J. Br. Interplanetary Soc. 56, 250 (2003).

40. Phillips, M., Sabas, M. \& Greenberg, J. Increased pentane and carbon disulfide in the breath of patients with schizophrenia. J. Clin. Pathol. 46, 861 (1993).

41. Sehnert, S. S., Jiang, L., Burdick, J. F. \& Risby, T. H. Breath biomarkers for detection of human liver diseases: preliminary study. Biomarkers 7, 174 (2002).

42. Eilers, P. H. C. A perfect smoother. Anal. Chem. 75(14), 3631-3636 (2003).

43 Karlovets, E. V., Gordon, I. E., Konnov, D., Muraviev, A. \& Vodopyanov, K. L. Dual-comb laser spectroscopy of CS2 near $46 \mu \mathrm{m}$. J. Quant. Spectrosc. Radiat. Transf. 256, 107269 (2020).

44. Zeng, Z., Altarawneh, M. \& Dlugogorski, B. Z. Atmospheric oxidation of carbon disulfide $\left(\mathrm{CS}_{2}\right)$. Chem. Phys. Lett. 669, 43 (2017).

\section{Acknowledgements}

We acknowledge support from the Office of Naval Research (ONR), Grant Number N00014-15-1-2659 and from the Defense Advanced Research Projects Agency (DARPA), Grant Number W31P4Q-15-1-0008. We thank Ekaterina Karlovets and Iouli Gordon for helping with the analysis of the $\mathrm{CS}_{2}$ spectra, and Peter Schunemann for providing nonlinear-optical OP-GaAs crystals.

\section{Author contributions}

A.V.M. constructed the experimental setup. D.K. developed the algorithm for data acquisition and processing. A.V.M. and D.K. carried out the measurements and analyzed the data. K.L.V. initiated and supervised the project; he also analyzed the data and wrote the paper.

\section{Competing interests}

The authors declare no competing interests.

\section{Additional information}

Correspondence and requests for materials should be addressed to K.L.V. 
Reprints and permissions information is available at www.nature.com/reprints.

Publisher's note Springer Nature remains neutral with regard to jurisdictional claims in published maps and institutional affiliations.

(c) (i) Open Access This article is licensed under a Creative Commons Attribution 4.0 International License, which permits use, sharing, adaptation, distribution and reproduction in any medium or format, as long as you give appropriate credit to the original author(s) and the source, provide a link to the Creative Commons licence, and indicate if changes were made. The images or other third party material in this article are included in the article's Creative Commons licence, unless indicated otherwise in a credit line to the material. If material is not included in the article's Creative Commons licence and your intended use is not permitted by statutory regulation or exceeds the permitted use, you will need to obtain permission directly from the copyright holder. To view a copy of this licence, visit http://creativecommons.org/licenses/by/4.0/.

(C) The Author(s) 2020 